\title{
Failure to Regulate: Counterproductive Recruitment of Top- Down Prefrontal-Subcortical Circuitry in Major Depression
}

\author{
Tom Johnstone, ${ }^{1}$ Carien M. van Reekum, ${ }^{2}$ Heather L. Urry, ${ }^{1,4}$ Ned H. Kalin,,${ }^{2,3}$ and Richard J. Davidson ${ }^{1,2,3}$ \\ ${ }^{1}$ Waisman Laboratory for Brain Imaging and Behavior, ${ }^{2}$ Department of Psychology, and ${ }^{3}$ Department of Psychiatry, University of Wisconsin-Madison, \\ Madison, Wisconsin 53705, and ${ }^{4}$ Department of Psychology, Tufts University, Medford, Massachusetts 02155
}

\begin{abstract}
Although depressed mood is a normal occurrence in response to adversity in all individuals, what distinguishes those who are vulnerable to major depressive disorder (MDD) is their inability to effectively regulate negative mood when it arises. Investigating the neural underpinnings of adaptive emotion regulation and the extent to which such processes are compromised in MDD may be helpful in understanding the pathophysiology of depression. We report results from a functional magnetic resonance imaging study demonstrating left-lateralized activation in the prefrontal cortex (PFC) when downregulating negative affect in nondepressed individuals, whereas depressed individuals showed bilateral PFC activation. Furthermore, during an effortful affective reappraisal task, nondepressed individuals showed an inverse relationship between activation in left ventrolateral PFC and the amygdala that is mediated by the ventromedial PFC (VMPFC). No such relationship was found for depressed individuals, who instead show a positive association between VMPFC and amygdala. Pupil dilation data suggest that those depressed patients who expend more effort to reappraise negative stimuli are characterized by accentuated activation in the amygdala, insula, and thalamus, whereas nondepressed individuals exhibit the opposite pattern. These findings indicate that a key feature underlying the pathophysiology of major depression is the counterproductive engagement of right prefrontal cortex and the lack of engagement of left lateral-ventromedial prefrontal circuitry important for the downregulation of amygdala responses to negative stimuli.
\end{abstract}

Key words: depression; prefrontal cortex; amygdala; emotion; regulation; fMRI

\section{Introduction}

Although abnormalities in the neural circuitry supporting adaptive regulation of emotion may play a decisive role in determining vulnerability to major depressive disorder (MDD) (Davidson, 2002), little research has been conducted to explicitly examine this possibility. Brain imaging studies in healthy individuals have identified a key corticolimbic circuit involved in the top-down regulation of affective subcortical circuitry. Increased left lateral prefrontal cortex (PFC) activation and decreased amygdala activation have been observed when individuals reappraise negative stimuli as less negative (Ochsner et al., 2002, 2004; Phan et al., 2005). Reappraising the affective meaning of negative emotional stimuli results in decreased magnitude of startle (Jackson et al., 2000), an objective indicator of negative emotion that involves the amygdala (Davis, 2006). An inverse relationship between amygdala and ventromedial PFC (VMPFC) activation has been observed when individuals reappraise the affective meaning of negative pictures (Urry et al., 2006). This inverse relationship reflects the inhibitory pathway from more dorsal and lateral regions of PFC to the amygdala and plays a role in learned extinc-

Received May 5, 2007; revised June 16, 2007; accepted July 5, 2007.

This work was supported by the National Institute of Mental Health. N.H.K. received grant support from WyethAyerst Pharmaceuticals. We thank Ron Fisher, Michael Anderle, and Sara Polis for assistance.

Correspondence should be addressed to Tom Johnstone, Waisman Laboratory for Brain Imaging and Behavior, 1500 Highland Avenue, Madison, WI 53705. E-mail: itjohnstone@wisc.edu.

D0I:10.1523/JNEUROSCI.2063-07.2007

Copyright $\odot 2007$ Society for Neuroscience $\quad$ 0270-6474/07/278877-08\$15.00/0 tion (Milad and Quirk, 2002). Individuals who show increased VMPFC and decreased amygdala activation during reappraisal also show steeper, more adaptive diurnal patterns of daily cortisol (Urry et al., 2006), suggesting that the capacity to successfully downregulate limbic circuitry has implications for well being in daily life.

Brain imaging studies of depressed individuals have demonstrated abnormal patterns of activation in prefrontal brain regions thought to contribute to the regulation of affect (Mayberg et al., 1997; Brody et al., 2001; Kennedy et al., 2001; Liotti et al., 2002; Davidson et al., 2003; Phillips et al., 2003; Keedwell et al., 2005), including hypoactivation in ventral and medial regions of PFC. One emotion regulation study (Beauregard et al., 2006) reported increased activation in dorsal anterior cingulate cortex (ACC) in depressed compared with healthy individuals when decreasing emotional responses to sad films but found no group differences in more anterior and lateral regions of PFC that have been found previously in studies of reappraisal.

To ascertain the extent to which depressed individuals engage prefrontal regions in the top-down regulation of subcortical affective circuitry, specifically the amygdala, we used functional magnetic resonance imaging (fMRI) to measure brain activation in 21 depressed individuals and an age-matched group of nondepressed controls. To probe prefrontal-amygdala regulatory circuitry, we chose an affective reappraisal task through which we previously observed an inverse relationship between activation in VMPFC and the amygdala (Urry et al., 2006), thought to reflect 
top-down regulatory influence of PFC on amygdala function. Pupil dilation provided an objective measure of autonomic arousal associated with effortful reappraisal. We predicted that during the condition in which subjects reappraise stimuli as less negative, patients with MDD would fail to show the adaptive reciprocal coupling between VMPFC and amygdala and would show abnormalities in the recruitment of lateral PFC regions compared with controls. We also predicted that for controls, activation in subcortical regions associated with negative affect, specifically the amygdala, would show an inverse relationship with effort-related pupil-dilation but expected that this relationship would be absent or positive in depressed patients.

\section{Materials and Methods}

Participants. Twenty-one medication-free, right-handed adults satisfying Diagnostic and Statistical Manual of Mental Disorders, Ed IV (DSMIV) criteria for unipolar major depressive disorder (age range, 19-53 years; mean age, 33 years; SD, 12; eight males) were compared with an age and sex-matched group of 18 right-handed controls (age range, 20-60 years; mean age, 28 years; SD, 12; seven males; age difference between groups not significant, $F_{(1,37)}=1.76 ; p=0.18$ ). All subjects were recruited via the use of flyers posted in a large number of public places (shops, libraries, etc.) around the Madison, WI metropolitan area. Depressed subjects had depressive symptoms for at least 1 month before their screening visit and a score of at least 18 on the Hamilton Rating Scale for Depression (HAM-D) (Hamilton, 1960) at screening and the first fMRI scan (mean HAM-D \pm SD depressed, $21 \pm 2.5$; controls, $0.5 \pm$ $0.6)$. In addition to standard MRI compatibility criteria, subjects were screened for and excluded if they met DSM-IV criteria for alcohol or drug abuse or dependence, other DSM-IV Axis I or Axis II diagnoses, had a personal or family history of bipolar disorder, or were using any medications that affect CNS function. In addition to the HAM-D, all participants completed the Hamilton Rating Scale for Anxiety (HAM-A) (Hamilton, 1959), Behavioral Inhibition System/Behavioral Activation System (BIS/BAS) scales (Carver and White, 1994), and the Penn State Worry Questionnaire (Meyer et al., 1990). Only the data from BIS/BAS scales are presented here, because they are the only scales that showed correlations with the brain imaging data. Additional details on inclusion and exclusion criteria and history of depressive episodes are given in the supplemental material (available at www.jneurosci.org). This research was approved by the University of Wisconsin-Madison Health Sciences Institutional Review Board, and all participants provided written informed consent.

Experimental task. The experimental task was a variant of that used previously in our laboratory with normal subjects (Urry et al., 2006) and similar to the tasks used in other recent studies (Jackson et al., 2000; Schaefer et al., 2002; Ochsner et al., 2004). Subjects were scanned while viewing a sequence of 72 emotionally positive and 72 negative pictures taken from the International Affective Picture System (IAPS) (Lang et al., 2005). Negative pictures were selected according to the IAPS norms to be both unpleasant ( 1 , most unpleasant, to 9 , most pleasant; $M=2.95$; SD, 0.87 ) and arousing $(1$, least arousing, to 9 , most arousing; $M=5.44 ; \mathrm{SD}$, $0.80)$, whereas positive images were pleasant $(M=7.13$; SD, 0.62$)$ and arousing $(M=5.28 ; \mathrm{SD}, 0.58)$. Stimuli were presented using E-Prime software (Psychology Software Tools, Pittsburgh, PA) via a fiber-optic goggle system (Avotec, Stuart, FL) with a screen resolution of $800 \times 600$ pixels. A $1 \mathrm{~s}$ fixation cross coupled with a tone oriented subjects to the upcoming trial, after which each image was presented for $10 \mathrm{~s}$, followed by a $6 \mathrm{~s}$ blank screen. To ensure participants remained attentive to the task, at the onset of each picture, subjects had to judge whether the image was positive or negative and respond with an appropriate button press on a two-button response pad. Four seconds into the presentation of each picture, an audio prompt instructed the participant to either increase ("enhance") or decrease ("suppress") their emotional response to the picture or to continue to "attend" to the picture.

Participants were trained during a previous session while positioned inside a mock scanner on the use of re-appraisal strategies to re-evaluate the images as more or less emotional (Jackson et al., 2000; Urry et al.,
2006). For the increase condition, participants were trained to either imagine themselves or a loved one experiencing the situation being depicted or imagine a more extreme outcome than the one depicted (e.g., in response to a picture of a ferocious dog, a participant might imagine that the dog's leash broke and the dog is going to bite them). Conversely, for the decrease condition, individuals were trained to either view the situation as fake or unreal or imagine that the situation being depicted had a different outcome than the one suggested (e.g., victims of a car accident survived and healed well). Alternatively, on attend trials, participants were instructed to maintain their attention to the picture without changing their negative affective experience. Simulated scanner sounds and task instructions were piped through earbud headphones during this training session. The training was succeeded by follow-up queries to ensure that participants were using the strategies as instructed and reported being able to perform the task.

In the subsequent experimental session, there were 20 repetitions of each regulation condition and 12 repetitions of the attend condition for each picture valence, evenly distributed over six scans, each lasting $380 \mathrm{~s}$. The order of positive and negative images and the three regulation instructions was pseudorandomized. Given the particular relevance for depressed subjects of downregulating activation in subcortical affective circuitry in response to negative stimuli, as well as our a priori hypotheses based on previous research, in this report, we present the data for the decrease-attend contrast in response to negative images.

Behavioral measures. Previous reports on top-down affective regulation have used self-reported ratings of valence and/or arousal after each trial, which are prone to demand characteristics and are reliant on accurate recall of the feelings experienced during regulation. Furthermore, requesting such reports engages subjects in a secondary self-reflective task that is likely to contaminate brain imaging results, leading to activation in brain areas potentially distinct from those actually involved in regulation (e.g., areas of the medial PFC) (Gusnard et al., 2001; Johnson et al., 2002). Rather than using self-reports of affect at the end of each trial, we measured the relative changes to pupil diameter throughout the task. This provides an objective, unobtrusive measure of autonomic arousal, with pupil constriction driven primarily by the parasympathetic branch of the autonomic nervous system (ANS), and pupil dilation primarily reflecting activity of the sympathetic branch (Loewenfeld, 1993). Pupil dilation is thus a component of the normal affective autonomic response to negatively valenced stimuli (Bernick and Overlander, 1968) but is also an indicator of increased cognitive and attentional load during effortful top-down regulation (Kahneman and Beatty, 1966; Siegle et al., 2003; Ohira et al., 2006).

To assess autonomic arousal associated with effortful reappraisal, we measured the extent to which the pupil dilated during the active reappraisal period of each stimulus trial. Based on our previous research showing pupil dilation to be a sensitive index of the cognitive effort during reappraisal in healthy individuals (Urry et al., 2006; van Reekum et al., 2007), we predicted that pupil dilation during the reappraisal period would be greater for increase and decrease trials than for attend trials. Additionally, we predicted that in controls, the decrease-attend pupil dilation difference would show an inverse correlation with decrease-attend brain activation in the amygdala, because with increasing effort, amygdala activation would be reduced (Urry et al., 2006). In depressed patients, we predicted either no relationship between decrease-attend pupil dilation and amygdala activation or possibly a positive correlation. The latter finding would be the case if pupil dilation in depressed individuals reflected emotional arousal resulting from unsuccessful attempts to reappraise negative stimuli.

A further consideration was the possibility that any observed differences between groups in regulation-associated brain activation might reflect depressed individuals paying more or less attention to the pictures than control subjects. To assess this possibility, we used the pupil data to compare the proportion of the reappraisal period that the eyes were clearly open across both experimental groups.

Pupil data acquisition and analysis. Horizontal pupil diameter data were acquired continuously at $60 \mathrm{~Hz}$ using an iView X system (v. 1.3.31) with a remote eye-tracking device (SensoMotoric Instruments, Teltow, Germany), which was interfaced with the fiber optic goggle system. Pupil 
data from four controls and six depressed individuals were not usable because of technical problems. Pupil dilation data were processed using algorithms written by Siegle et al. (2002) (unpublished MatLab code) with MatLab software (MathWorks, Natick, MA), modified in our laboratory. Blinks were identified and eliminated using local regression slopes and amplitude thresholds. Data were smoothed with a five-sample rolling average and linearly detrended over each scan run. For successive 500 $\mathrm{ms}$ bins in each trial, the proportion of time that the eye was open and mean pupil diameter were calculated.

Pupil values were then range-corrected to standardize according to the pretrial maximally dilated pupil diameter and the maximally constricted pupil diameter in the $2 \mathrm{~s}$ after picture onset [(current pupil diameter minimum pupil diameter $) /($ maximum pupil diameter - minimum pupil diameter)]. Data were averaged across a $5 \mathrm{~s}$ interval starting $1 \mathrm{~s}$ after instruction and continuing until picture offset (the reappraisal period). Data were then analyzed using mixed model GLM (subject as a random factor nested within the fixed factor group, and reappraisal as a withinsubject fixed factor).

Image acquisition. Images were collected on a General Electric 3 Tesla scanner (GE Medical Systems, Waukesha, WI) equipped with a standard clinical whole-head transmit-receive quadrature head coil. Functional images were acquired using a $\mathrm{T} 2^{\star}$-weighted gradient-echo, echo planar imaging (EPI) pulse sequence [33 sagittal slices, $4 \mathrm{~mm}$ thickness, $1 \mathrm{~mm}$ interslice gap; $64 \times 64$ matrix; $240 \mathrm{~mm}$ field of view (FOV); repetition time (TR)/echo time (TE)/Flip, $2000 \mathrm{~ms} / 30 \mathrm{~ms} / 60^{\circ}$; 190 whole-brain volumes per run]. A high-resolution T1-weighted anatomical image was also acquired (T1-weighted inversion recovery fast gradient echo; $256 \times$ 256 in-plane resolution; $240 \mathrm{~mm}$ FOV; $124 \times 1.1 \mathrm{~mm}$ axial slices).

Image analysis. Individual subject data were slice-time corrected, motion corrected, and analyzed in AFNI (Cox, 1996) using a GLM with a separate regressor for each trial type, six motion estimate covariates (Johnstone et al., 2006), and a second-order polynomial used to model the baseline and slow signal drift. Regressors consisted of a set of five sine basis functions to produce separate estimated hemodynamic response functions (HRFs) for each trial type. The estimated HRFs were converted to percentage signal change values, and within-subjects contrasts were calculated between the decrease and attend conditions for negative pictures (i.e., negative decrease - negative attend), averaged across time points corresponding to the peak hemodynamic response during the regulation period ( $8-14 \mathrm{~s}$ after stimulus onset). Contrasts were normalized to Talairach space using FLIRT (Jenkinson et al., 2002) and smoothed using a $6 \mathrm{~mm}$ full-width at half-maximum Gaussian filter. These smoothed contrast maps were then entered into a mixed-effects GLM with subject as a random factor nested in the fixed factor group to assess main reappraisal effects as well as group effects and group by reappraisal interactions. To assess functional connectivity of amygdala with ventromedial prefrontal cortex, we extracted the decrease-attend percentage signal change value from a Talairach-defined amygdala region of interest and used this as a covariate in a voxel-wise mixed-effects analysis. To test the relationship between ANS activity associated with the reappraisal task and brain activation, we included decrease-attend pupil dilation differences during the reappraisal period as a covariate in a voxel-wise mixed-effects analysis.

Group statistical analyses also included age as a covariate, which was not found to affect any of the results reported here. All statistical maps were thresholded at $p<0.01$, corrected for multiple comparisons using cluster-size thresholding based on Monte Carlo simulation. With this technique, the overall family-wise error rate (FWE) is controlled by simulating null data sets with the same spatial autocorrelation as found in the residual images and creating a frequency distribution of different cluster sizes. Clusters with a size that exceeds the minimum cluster size corresponding to the a priori chosen FWE are retained for additional analysis. This cluster-based method of thresholding, analogous to cluster-based thresholding using Gaussian Random Field Theory (Friston et al., 1994), is an alternative to voxel-based correction and is often more sensitive to activation when one can reasonably expect multiple contiguous activated voxels (Forman et al., 1995; Petersson et al., 1999). Individual mean contrast estimates for significant clusters were extracted for additional analysis using SPSS (SPSS, Chicago, IL). To explicitly test for hemi- spheric asymmetry, we reflected each cluster mask about the $x=0$ plane (the midline) and extracted contrast estimates from the resulting opposite hemisphere homologous clusters.

\section{Results}

\section{Group differences in pupillary measures of attention,} reappraisal effort, and arousal

To assess the possibility that differences between groups in reappraisal-associated brain activation might be because of differences in the attention being paid to the pictures, we compared the proportion of the reappraisal period that the eyes were open across both experimental groups. There were no main effects of reappraisal $\left(F_{(2,56)}=1.76 ; p=0.18\right)$ or group $\left(F_{(1,28)}=0.01 ; p=\right.$ $0.91)$ nor an interaction of group by reappraisal $\left(F_{(2,56)}=1.0 ; p=\right.$ 0.37 ) on the proportion of time that the eyes were open. Concentrating on only the decrease and attend conditions (the focus of this report), there was a trend for the proportion of time eyes were open to be lower for decrease than for attend $\left(F_{(1,28)}=3.73\right.$; $p=0.064$; mean \pm SE for attend, $0.747 \pm 0.005$; for decrease, $0.713 \pm 0.004)$ but no effect of group $\left(F_{(1,28)}=0\right)$ nor was there any group by reappraisal interaction $\left(F_{(1,28)}=0.52 ; p=0.48\right)$. Thus, both groups spent the same proportion of trials with their eyes open across all conditions.

To assess autonomic arousal associated with effortful reappraisal, we measured the extent to which the pupil remained dilated during the active reappraisal period of each stimulus trial. Replicating our previous work (Urry et al., 2006; van Reekum et al., 2007), pupil dilation showed a significant main linear effect of reappraisal $\left(F_{\text {linear }(1,28)}=17.7 ; p<0.001\right)$, with most sustained dilation for increase (relative proportional dilation \pm SE for controls, $0.001 \pm 0.027$; depressed, $-0.040 \pm 0.025$ ) than for decrease (controls, $-0.102 \pm 0.026$; depressed, $-0.0116 \pm 0.024$ ) and the least sustained dilation for the attend condition (controls, $-0.148 \pm 0.026$; depressed, $-0.140 \pm 0.024)$. There was no main effect of group on pupil dilation during reappraisal $\left(F_{(1,28)}=\right.$ $0.53 ; p=0.471)$, nor was there an interaction of group with reappraisal condition on pupil dilation $\left(F_{(2,56)}=0.41 ; p=\right.$ $0.667)$. Concentrating on only the decrease and attend conditions (the focus of this report), the results were also as predicted, with greater dilation for decrease than for attend $\left(F_{\text {linear }(1,28)}=3.1\right.$; $p=0.045)$ but no effect of group on pupil dilation $\left(F_{(1,28)}=0.01\right.$; $p=0.913)$ nor an interaction of group with reappraisal condition on pupil dilation $\left(F_{(1,28)}=0.28 ; p=0.602\right)$.

Together, these analyses indicate that both groups showed no differences in their level of attention to the task and amount of cognitive effort expended during reappraisal of the pictures.

\section{Effects of reappraisal on PFC activation}

Several brain regions showed greater activation in both depressed and control individuals in the decrease condition than in the attend condition. These areas of activation replicated previous findings obtained from nondepressed individuals (Ochsner et al., 2004; Phan et al., 2005) and included left lateral and ventrolateral PFC [left middle frontal gyrus (MFG), BA 8; left inferior frontal gyrus (IFG), BA 45/47] and right insula (BA 13) (Table 1). In addition, relatively less activation occurred during the decrease condition than during the attend condition in right inferior parietal lobule (BA 40) and the left superior temporal gyrus (BA 41). Tests of hemispheric asymmetry revealed that all of the activation clusters were significantly lateralized across both the control and depressed groups except for the right insula, which was not lateralized, and the left ventrolateral PFC cluster, which was lateralized in controls but not in the depressed subjects (Fig. 1) 
Table 1. Statistically significant clusters emerging from the voxel-wise mixed-effects GLM on the negative pictures decrease-attend blood oxygen level-dependent contrast estimates

\begin{tabular}{|c|c|c|c|c|c|c|}
\hline \multirow[b]{2}{*}{ Brain region } & \multirow[b]{2}{*}{$\operatorname{Max} Z$} & \multirow[b]{2}{*}{ Cluster size $\left(\mathrm{mm}^{3}\right)$} & \multicolumn{3}{|c|}{$\begin{array}{l}\text { Talairach coordinates of cluster } \\
\text { center }\end{array}$} & \multirow[b]{2}{*}{ Direction of effect } \\
\hline & & & $x$ & $y$ & $z$ & \\
\hline \multicolumn{7}{|l|}{ Patients and controls } \\
\hline IFG (BA 45,47) (depicted in Fig. 1) & 3.80 & 3264 & -47 & 24 & 0 & $\begin{array}{l}\text { Patients: } \mathrm{LH} \text {, decrease }>\text { attend; } R H \text {, decrease }>\text { attend } \\
\text { Controls: } \mathrm{LH} \text {, decrease }>\text { attend; } R H \text {, decrease }=\text { attend }\end{array}$ \\
\hline Left MFG (BA 8) & 4.26 & 5112 & -42 & 10 & 44 & Decrease $>$ attend \\
\hline Right insula (BA 13) & 3.93 & 2936 & 44 & 14 & -1 & Decrease $>$ attend \\
\hline Right inferior parietal lobule (BA 40) & 4.33 & 4576 & 39 & -55 & 41 & Decrease $<$ attend \\
\hline Left superior temporal gyrus (BA 41) & 3.34 & 2088 & -57 & -18 & 7 & Decrease $<$ attend \\
\hline \multicolumn{7}{|l|}{ Patients $>$ controls } \\
\hline MFG (BA 10) (depicted in Fig. 2) & 3.62 & 1504 & 32 & 46 & 25 & $\begin{array}{l}\text { Patients: } \mathrm{RH}, \text { decrease }>\text { attend; } \mathrm{LH}: \text { decrease }=\text { attend } \\
\text { Controls: } \mathrm{RH} \text {, decrease }<\text { attend; } \mathrm{LH}, \text { decrease }=\text { attend }\end{array}$ \\
\hline
\end{tabular}

LH, Left hemisphere; RH, right hemisphere.
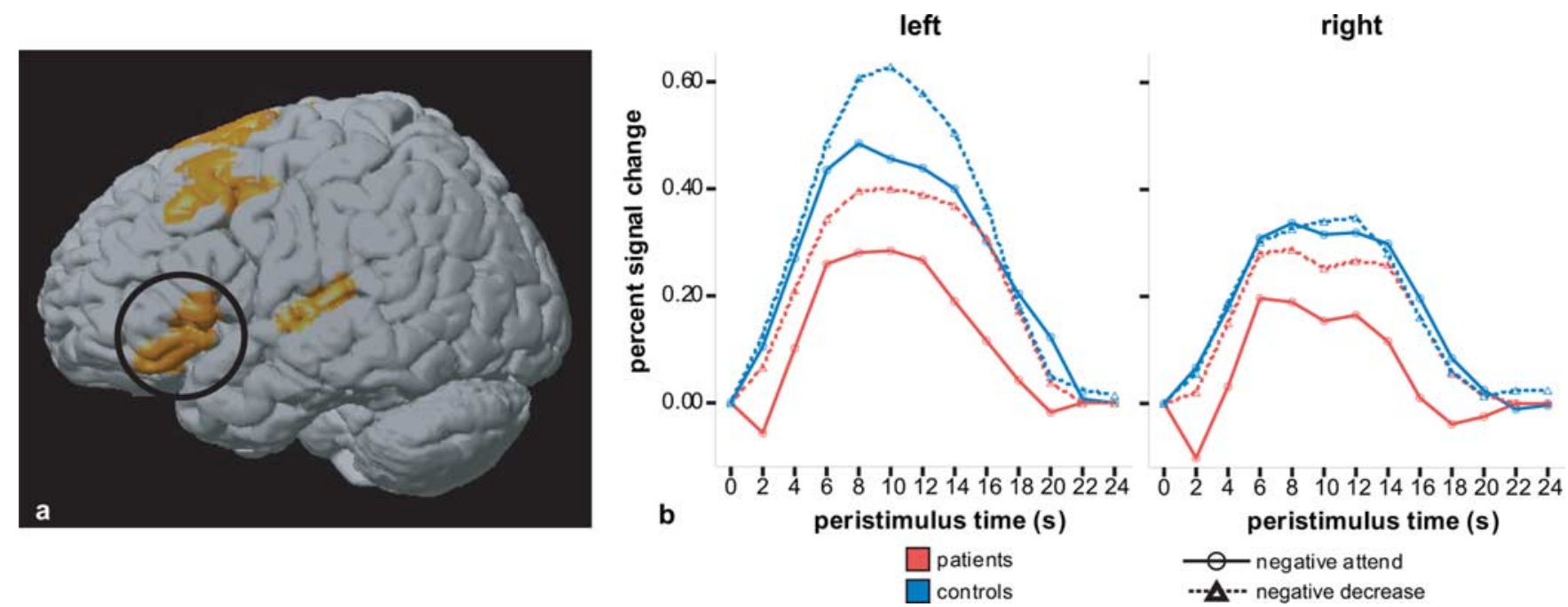

Figure 1. Activation in IFG when downregulating emotional responses to negative pictures versus attending to negative pictures. $\boldsymbol{a}$, Cluster in left IFG shows decrease-attend activation for healthy controls and depressed individuals. Activation in the right IFG is attributable to the depressed group but not the control group having decrease-attend activation in this region. Talairach coordinates: $x \pm 47, y=24, z=0, B A 45 / 47 . b$, Mean peristimulus plots of estimated BOLD responses to negative pictures in the attend condition (solid line) and the decrease condition (dashed line). The control group is shown in blue, and the depressed group is shown in red. The activation was left-lateralized in controls but bilateral in the depressed group (group $\times$ regulation $\times$ hemisphere interaction for cluster mean, $\left.F_{(1,37)}=4.26 ; p=0.046\right)$.

(group $\times$ reappraisal $\times$ hemisphere interaction for cluster mean, $F_{(1,37)}=4.26 ; p=0.046$; Talairach coordinates, $x=-47, y=24$, $z=0)$. In this latter cluster, during the decrease condition, controls showed increased activation in left ventrolateral PFC $\left(F_{(1,17)}=12.15 ; p=0.003\right)$ but not in right ventrolateral PFC $\left(F_{(1,17)}=0.08 ; p=0.780\right)$, whereas depressed individuals showed increased activation bilaterally (left, $F_{(1,20)}=7.02$, $p=0.023$; right, $\left.F_{(1,20)}=7.68, p=0.012\right)$.

A cluster in the right lateral PFC (right middle frontal gyrus; BA 10) showed a significant group $\times$ reappraisal $\times$ hemisphere interaction (Fig. 2) (for cluster mean, $F_{(1,37)}=8.01, p=0.007$; Talairach coordinates, $x=32, y=46, z=25)$. In this right lateral PFC region, controls showed less activation in the decrease condition, relative to the attend condition $\left(F_{(1,17)}=9.92 ; p=0.006\right)$, whereas depressed patients showed greater activation in the decrease condition than the attend condition $\left(F_{(1,20)}=12.80 ; p=\right.$ $0.002)$. Neither group showed a significant effect of reappraisal in the same region of the left hemisphere (controls, $F_{(1,17)}=0.01$, $p=0.926$; depressed, $\left.F_{(1,20)}=0.91, p=0.352\right)$. This pattern of prefrontal activation thus indicates a greater relative recruitment of right prefrontal cortex during reappraisal in major depression.

\section{Amygdala-PFC connectivity}

Consistent with our previous study in healthy individuals (Urry et al., 2006), we found no decrease-attend reappraisal effect for either the control group or depressed group on activation in the amygdala, a principal subcortical structure that is involved in the generation of emotional responses. Our previous work had, however, demonstrated an inverse relationship between amygdala and VMPFC activation when nondepressed individuals reappraised negative pictures as less negative, reflecting individual differences in the ability to engage top-down prefrontal regulatory circuitry. Based on this previous finding as well as current knowledge of the connectivity of VMPFC to both lateral PFC and the amygdala (Barbas,1995; Ongur and Price, 2000; Maren and Quirk, 2004) and the role of medial PFC in downregulation of amygdala function (Amaral and Price, 1984; Quirk et al., 2003; Phelps et al., 2004), we used the decrease-attend signal contrast in amygdala for each group as a separate covariate in a voxel-wise mixed-effects GLM analysis and assessed the between-group difference in the covariate fit coefficients. This yielded a corrected voxelwise map of regions in the brain in which the correlation between amygdala activation and local brain activation differed significantly 


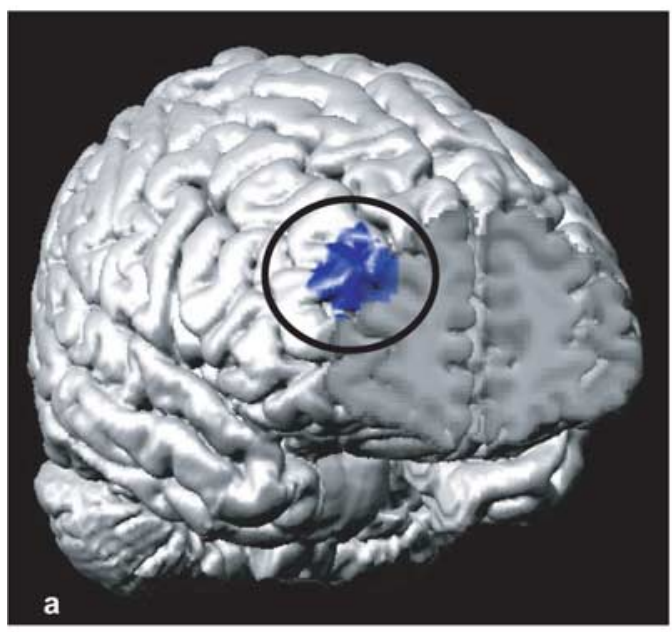

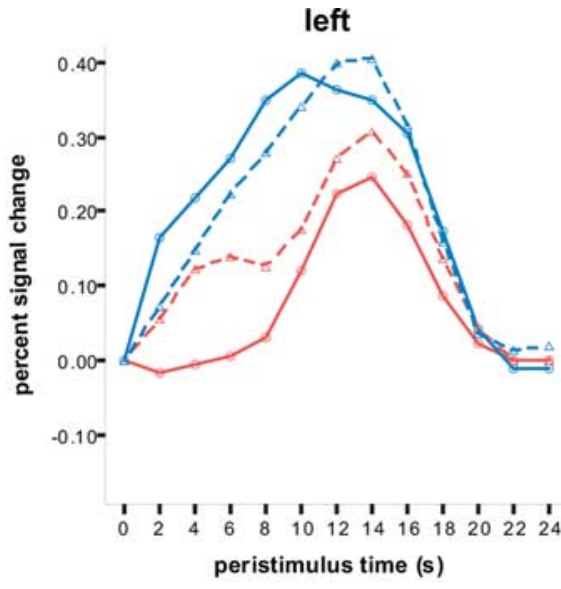

b

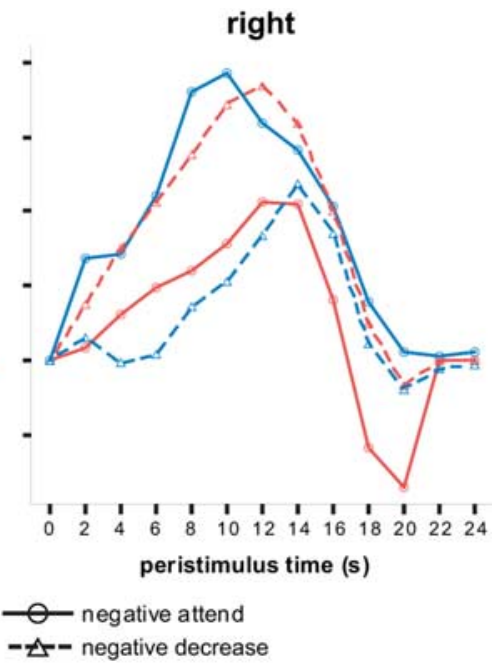

Figure 2. Cluster in the right MFG showing a group by regulation interaction. $\boldsymbol{a}$, Cluster location Talairach coordinates: $x=32, y=46, z=25$, BA 10. $\boldsymbol{b}$, Peristimulus time plots of estimated B0LD responses as a function of group and regulation condition. In the right $\mathrm{MFG}$, controls showed a decrease in activation when decreasing responses to negative pictures (relative to attending), whereas the depressed individuals showed an increase in activation when decreasing their responses (group $\times$ condition $\times$ hemisphere interaction for cluster mean: $F_{(1,37)}=8.01 ; p=0.007$ ).
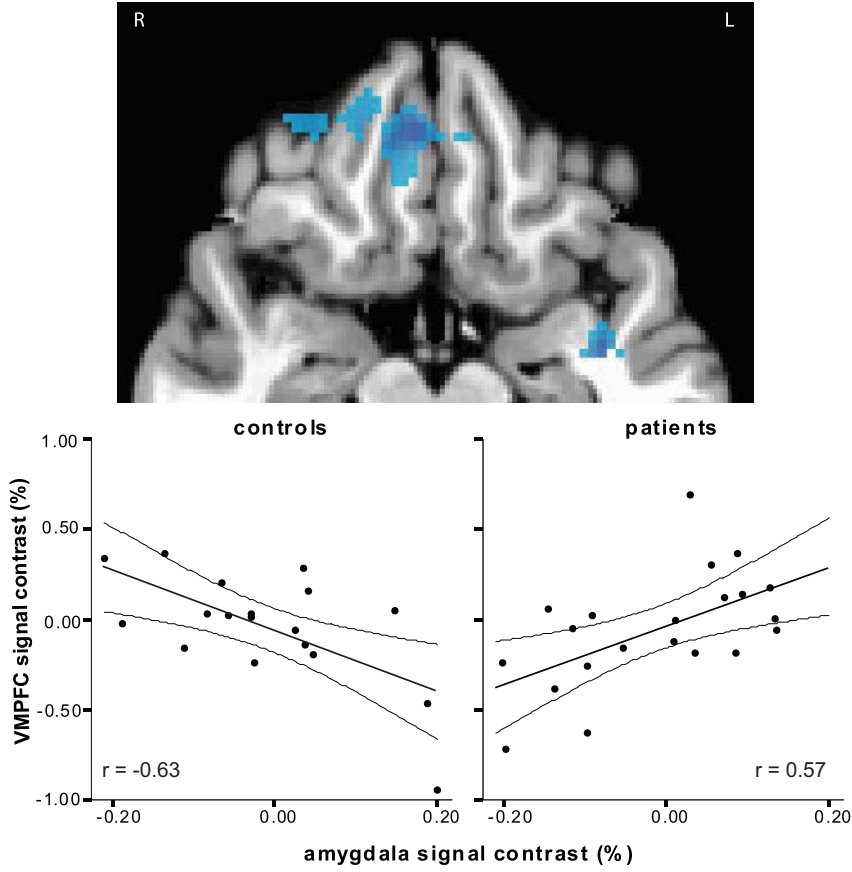

Figure 3. Cluster in VMPFC showing significantly different decrease-attend correlation with amygdala for controls versus the depressed group. VMPFC activation correlated negatively with amygdala in controls $(r=-0.63 ; p=0.005)$ but correlated positively for the depressed group $(r=$ $0.57 ; p=0.007 ;$ Talairach coordinates, $x=7, y=39, z=-11, \mathrm{BA} 11 / 32$ ). R, Right; L, left.

between groups. We hypothesized that in contrast to our previous study and the controls, depressed patients would fail to show an inverse association between activation in these regions.

As predicted, the analysis revealed a cluster in $\operatorname{VMPFC~(Fig.~3)~}$ (Talairach coordinates, $x=7, y=39, z=-11$; BA 11/32) that showed a significantly different correlation with amygdala in the control versus depressed individuals $(Z=-3.9729 ; p<0.001)$. Replicating our previous finding, there was a negative correlation between the decrease-attend contrast in amygdala and VMPFC in controls $(r=-0.63 ; p=0.005)$, such that nondepressed individuals who showed the greatest VMPFC activation when reappraising negative stimuli also showed the lowest level of activation in the amygdala. In the depressed group, however, there was a positive correlation in decrease-attend activation between these two brain regions $(r=0.57 ; p=0.007)$, indicating that in depressed individuals who showed the greatest VMPFC activation, the amygdala also showed greater activation.

We previously demonstrated that the VMPFC plays a crucial role in mediating the effect of other regions of PFC on the amygdala (Urry et al., 2006). Consistent with this previous finding, activation in the VMPFC cluster was found to be a significant mediator of an effect of left (but not right) ventrolateral PFC on amygdala in controls (test of mediation $Z=-1.86, p=0.03$, one-tailed; $r$ [IFG-VMPFC] $=0.48, r$ IFG-AMYG] $=0.12$, $r[\mathrm{VMPFC}-\mathrm{AMYG}]=-0.63)$. There was no such mediational effect for the depressed group $(Z>0 ; r[$ IFG-VMPFC $]=0.60$, $r[$ IFG-AMYG $]=0.54, r[$ VMPFC-AMYG $]=0.57)$. This analysis provides additional evidence that, in controls, the left ventrolateral PFC exerts an inhibitory effect on the amygdala via the VMPFC during the active reappraisal of negative stimuli, whereas this circuit is dysfunctional in major depression (Fig. 4).

Correlations between self-reported emotional disposition and activation in these clusters further buttress the role of these clusters in adaptive regulation. For the depressed group, there was a negative correlation between scores on the BAS scale, a measure of behavioral approach, and amygdala decrease-attend activation, such that depressed individuals with higher amygdala activation scored lower on the BAS scale $(r=-0.55, p=0.01$; correlation not significant for control subjects, $r=-0.23, p=$ 0.35 ; mean $\pm \mathrm{SD}$; BAS depressed, $33.0 \pm 4.7$, controls, $42.6 \pm 4.6$; difference between groups, $t(36)=6.3 ; p<0.001)$. Depressed individuals scoring higher on the BIS scale, a measure of behavioral inhibition, tended to show lower decrease-attend activation in the left ventrolateral PFC cluster $(r=-0.39, p=0.08$; for controls, $r=0.19, p=0.45$; mean $\pm \mathrm{SD}$; BIS depressed, $24.1 \pm$ 2.8 , controls, $18.2 \pm 3.3$; difference between groups, $t(37)=6.2$; $p<0.001$ ), suggesting deficient recruitment of this region by those reporting the highest levels of behavioral inhibition, adding further support to the purported role of this region in adaptive downregulation of the amygdala via the VMPFC. More generally, these findings are consistent with previous research demonstrating that behavioral inhibition and approach differentiate be- 


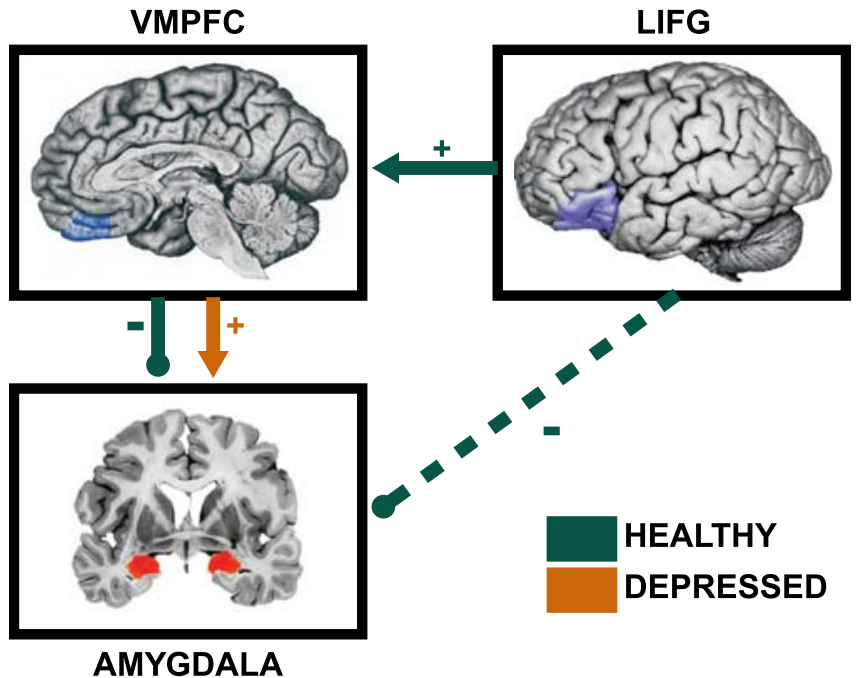

Figure 4. Schematic depicting brain regions that showed a significantly different association with amygdala in the control versus depressed individuals. In healthy controls, there was a negative correlation between the decrease-attend contrast in VMPFC and amygdala $(r=$ $-0.63 ; p=0.005$ ), such that nondepressed individuals who showed the greatest VMPFC activation when downregulating their emotional responses, relative to attending to the pictures, also showed the lowest amygdala activation. The VMPFC was found to be a significant mediator of the association between left IFG (LIFG) and amygdala in controls $(Z=-1.86 ; p=$ 0.03 , one-tailed). In the depressed group, there was a positive correlation in activation between the VMPFC and amygdala $(r=0.57 ; p=0.007)$ when decreasing their emotional response relative to the attend condition, with no mediational effect of $\operatorname{VMPFC}(Z>0)$.

tween depressed versus nondepressed individuals and are predictive of depression symptom severity and outcome over an extended 8 month period (Kasch et al., 2002).

\section{Brain activation associated with changes in pupil dilation}

To assess the relationship between brain activation and the level of associated autonomic arousal while reappraising negative stimuli, we examined the degree of pupil dilation during the reappraisal period. We included the decrease-attend pupil dilation contrast for each group as a separate covariate in a mixed-effects GLM predicting the decrease-attend BOLD contrast and assessed the between-group difference in the covariate fit coefficients. This yielded a corrected voxel-wise map of regions in the brain in which the correlation between pupil dilation and brain activation differed significantly between groups.

This analysis yielded four significant clusters (Fig. 5): left insula (extending into left putamen), right insula (extending into right inferior frontal gyrus), left amygdala (extending superiorly into lentiform nucleus), and a large midbrain/thalamus cluster. Across all four clusters, the pattern of results was the same, with controls showing a significant negative correlation of pupil dilation difference with decrease-attend activation and depressed individuals showing a significant positive correlation of pupil dilation difference with decrease-attend contrast (differences between correlation for controls and correlation for depressed are significant; all $z>3.97 ; p<0.001$ ). The result for the controls is consistent with the notion that individuals exerting relatively more cognitive effort while reappraising negative stimuli, as reflected in greater effort-related pupil dilation, showed a relative decrease in activation in the amygdala and insula, both brain regions known to be involved in the generation and experience of negative emotion. The result for patients was precisely opposite: depressed patients show a positive correlation between activation in core emotion circuitry and pupil dilation.
This pattern of data suggests that effortful attempts by depressed individuals to reappraise negative stimuli as less negative are counterproductive and lead to an increase rather than a decrease in the magnitude of the emotional response, as reflected in activation of amygdala, insula, and thalamic components of emotion circuitry. The presence of a positive correlation between amygdala activation and VMPFC in depressed patients during reappraisal of negative images, two regions that in the control group and previous studies are inversely coupled, and a lack of mediation by VMPFC of ventrolateral PFC effects on amygdala activation for the depressed individuals, supports this conclusion.

\section{Discussion}

The prefrontal cortex is thought to play a crucial role in the topdown regulation of subcortical affective circuitry. The ventrolateral PFC in particular is known to play a role in the learning of new affective stimulus associations and the reversal of previously learned valence-stimulus contingencies (Rolls et al., 1996; Morris and Dolan, 2004). Using a reappraisal task that has been shown previously to engage top-down regulatory PFC circuitry (Jackson et al., 2000; Urry et al., 2006), we demonstrate decrease-attend ventrolateral PFC activation in both healthy controls and depressed individuals that is consistent with such a functional role for this region of PFC. Increased activation in a more dorsal region of left lateral PFC while reappraising negative stimuli relative to attending to negative pictures was also observed in both experimental groups, a result consistent with previous research that has implicated the more lateral regions of PFC in the appraisal and reappraisal of affective stimuli.

Previous research with depressed individuals has shown less activation in left lateral regions of the PFC but greater activation in the right PFC than in healthy controls in response to sad autobiographical scripts (Keedwell et al., 2005). Greater relative left prefrontal brain activity is related to a greater disposition to approach-related, positive effect (Tomarken et al., 1992) and a greater ability to regulate negative effect (Jackson et al., 2003), whereas greater relative right prefrontal activation has been associated with withdrawal-related, often negative effect (Davidson, 1995). Although we did not find any difference in left dorsolateral PFC activation between depressed and healthy individuals in this study, we did find functional hemispheric asymmetries that accord with these previous findings. Thus, depressed individuals in this study showed greater activation in right lateral and ventrolateral PFC when reappraising negative images than did the healthy controls. This increased activation in the right PFC of depressed individuals and the absence of left-lateralized activation as seen in controls indicates an inappropriate or inefficient engagement of prefrontal regulatory circuitry.

The VMPFC is thought to be a key part of this regulatory circuitry, acting as a link between lateral prefrontal circuits involved in reappraising the emotional significance of affective stimuli and subcortical circuits crucial for the generation of emotional responses. In nondepressed individuals, we replicated our previous finding (Urry et al., 2006) of an inverse relationship between activation in the VMPFC and the amygdala. Furthermore, the VMPFC mediates the association between left ventrolateral PFC and amygdala activation during the downregulation condition. We observed the opposite relationship between VMPFC and amygdala activation in the depressed group and the absence of any mediation effect. This result is particularly noteworthy, because although there are no direct neuronal connections between lateral PFC and the amygdala (Ghashghaei and Barbas, 2002), the medial prefrontal cortex, including the area of VMPFC reported here, BA 11, is directly linked to the amygdala and is also connected to dorsal and lateral PFC (Barbas,1995; 


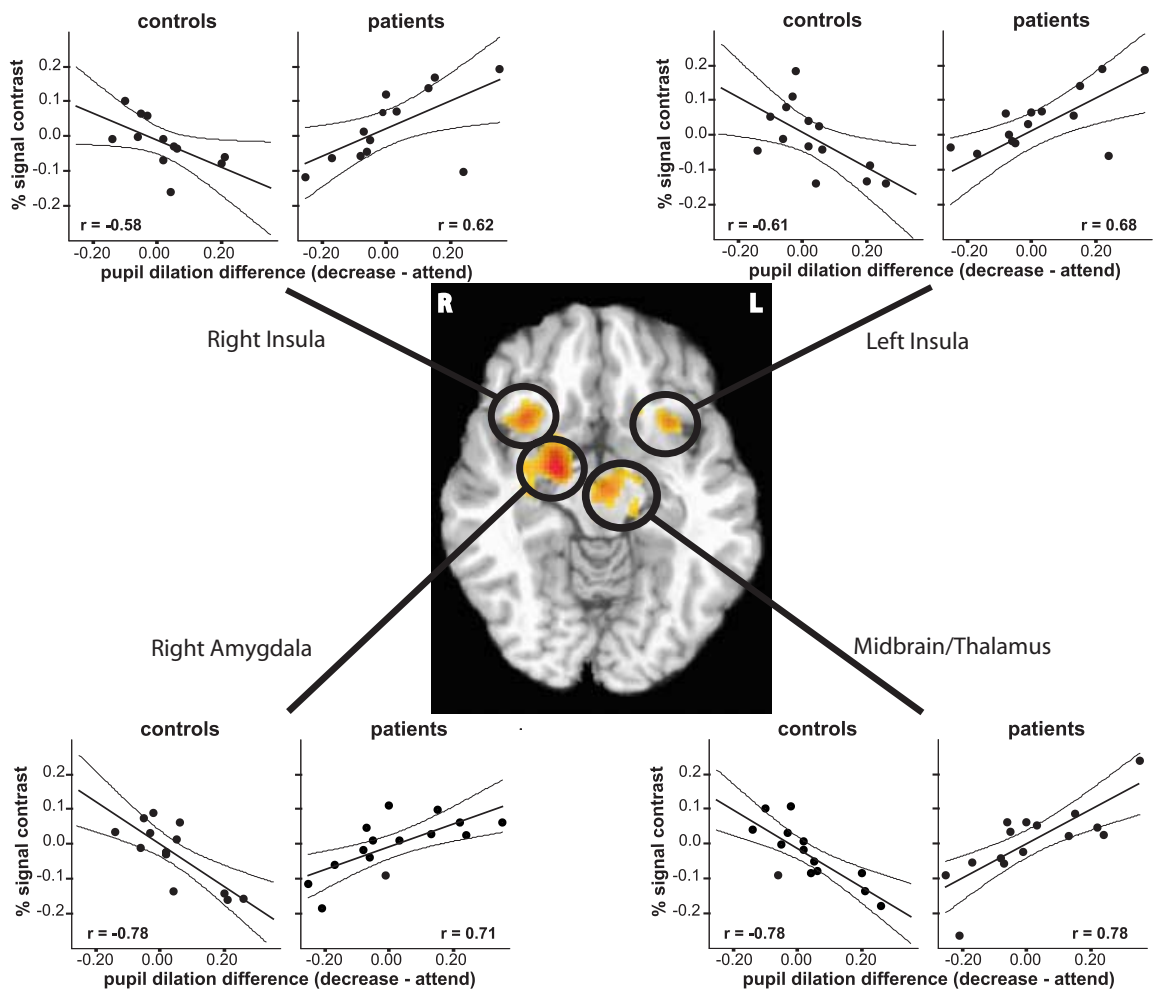

Figure 5. Regions showing a significant group difference in the correlation between pupil dilation (decrease-attend) and brain activation (decrease-attend) while reappraising negative pictures. Across all clusters, the same pattern was evident, with a negative correlation in controls and a positive correlation in the depressed group. R, Right; L, left.

Ongur and Price, 2000; Maren and Quirk, 2004). Furthermore, the medial PFC has been implicated in downregulation of amygdala function (Amaral and Price, 1984; Quirk et al., 2003; Phelps et al., 2004). Our data suggest that the role of VMPFC as an inhibitory link between left ventrolateral PFC and the amygdala is compromised in depression, likely because of the inappropriate engagement of right PFC circuitry in depressed individuals. Furthermore, our previous research has shown that in healthy individuals, the engagement of VMPFC-amygdala regulatory circuitry predicts steeper, more adaptive diurnal patterns of daily cortisol (Urry et al., 2006), a finding that has important implications for well being in daily life. Flattening of the diurnal profile of cortisol has been observed in individuals with MDD (den Hartog et al., 2003). Our research points to a possible mechanism that may underlie this aspect of depression. Future research will be required to explicitly test this hypothesis.

The association between pupil dilation and brain activation in this study provided additional evidence for the compromised functioning of top-down regulation of limbic circuitry in depression. In controls, increased effort to reappraise negative stimuli as reflected in greater pupil dilation was associated with decreased activation in amygdala and insula, two crucial sites for the generation of emotional responses. These findings replicate the inverse association between left amygdala and pupil dilation in our previous work (Urry et al., 2006) and extend it by showing the inverse relationship with the insula, a region also involved in autonomic output and bodily responses and often activated in response to emotional stimuli. These data strongly suggest that more effort expended by healthy individuals when reappraising is associated with successful downregulation of these limbic system regions. In depressed subjects, however, pupil dilation was strongly positively correlated with activation in these same regions. This finding suggests that the effortful attempt to reappraise negative stimuli was ineffective or counterproductive in the depressed group. In such cases where the prefrontal downregulation of affective subcortical circuitry is compromised in depression, it is likely that amygdala responses to negatively valenced images drive sympathetic autonomic responses, giving rise to the positive correlation between amygdala BOLD responses and pupil dilation.

The data from this study suggest that the inappropriate engagement of lateral PFCVMPFC-amygdala inhibitory circuitry during efforts to reappraise negative emotional stimuli might be one of the key factors underlying the pathophysiology of major depression. It should be noted, however, that the finding of VMPFC downregulating amygdala response is likely intimately linked to the top-down nature of the task. Other studies that have used tasks that emphasize bottom-up mechanisms involved in the generation of responses to affective stimuli have found quite different results, with a positive link between amygdala response and VMPFC/subgenual ACC activation (Pezawas et al., 2005). This should not be seen to contradict the results from this, or previous reappraisal studies. The connections between amygdala and VPMFC are bidirectional (Ghashghaei et al., 2007). We propose that the amygdala can "drive" VMPFC in a bottom-up affective reactivity task but be downregulated by more dorsal and lateral portions of the PFC via the VMPFC in a top-down reappraisal task. Indeed, striking an optimal balance between such bottom-up and top-down influences in a given emotional situation is likely to be crucial for the individual to respond adaptively. Evidence from this study suggests that in major depression, this balance is upset, with the amygdala exerting its influence on prefrontal cortex in a dysregulated manner.

The findings obtained in this study are based on a very short period of training in cognitive reappraisal. Having established that individuals with depression show abnormalities in the recruitment of prefrontal resources and in connectivity between prefrontal regions and the amygdala during such tasks, it will be essential to study the impact of cognitive therapy and other related forms of reappraisal training on the neural circuitry of emotion regulation. It may well be that those depressed individuals who show the greatest pupil diameter and corresponding increases in activation in the amygdala and insula during the reappraisal condition are individuals likely to exhibit less improvement in response to cognitive therapy. Thus, neural activity in response to this task could potentially be used as a predictor of treatment response to cognitive therapy if this prediction were to be supported in future research. However, more enduring training than was accomplished in this study may normalize this circuitry in depressed patients. This issue requires study in future research.

\section{References}

Amaral DG, Price JL (1984) Amygdalo-cortical projections in the monkey (Macaca fascicularis). J Comp Neurol 230:465-496.

Barbas H (1995) Anatomic basis of cognitive-emotional interactions in the primate prefrontal cortex. Neurosci Biobehav Rev 19:499-510.

Beauregard M, Paquette V, Levesque J (2006) Dysfunction in the neural circuitry of emotional self-regulation in major depressive disorder. NeuroReport 17:843-846. 
Bernick N, Overlander M (1968) Effect of verbalization and two different modes of experiencing on pupil size. Percept Psychophys 3:327-330.

Brody AL, Saxena S, Mandelkern MA, Fairbanks LA, Ho ML, Baxter LR (2001) Brain metabolic changes associated with symptom factor improvement in major depressive disorder. Biol Psychiatry 50:171-178.

Carver CS, White TL (1994) Behavioral inhibition, behavioral activation, and affective responses to impending reward and punishment: the BIS/ BAS scales. J Pers Soc Psychol 67:319-333.

Cox RW (1996) AFNI: software for analysis and visualization of functional magnetic resonance neuroimages. Comput Biomed Res 29:162-173.

Davidson RJ (1995) Cerebral asymmetry, emotion and affective style. In: Brain asymmetry (Davidson RJ, Hugdahl K, eds), pp 361-387. Cambridge, MA: MIT.

Davidson RJ (2002) Anxiety and affective style: role of prefrontal cortex and amygdala. Biol Psychiatry 51:68-80.

Davidson RJ, Irwin W, Anderle MJ, Kalin NH (2003) The neural substrates of affective processing in depressed patients treated with venlafaxine. Am J Psychiatry 160:64-75.

Davis M (2006) Neural systems involved in fear and anxiety measured with fear-potentiated startle. Am Psychol 61:741-756.

den Hartog HM, Nicolson NA, Derix MM, van Bemmel AL, Kremer B, Jolles J (2003) Salivary cortisol patterns and cognitive speed in major depression: a comparison with allergic rhinitis and healthy control subjects. Biol Psychol 63:1-14.

Forman SD, Cohen JD, Fitzgerald M, Eddy WF, Mintun MA, Noll DC (1995) Improved assessment of significant activation in functional magnetic resonance imaging (fMRI): use of a cluster-size threshold. Magn Reson Med 33:636-647.

Friston KJ, Worsley KJ, Frackowiak RSJ, Mazziotta JC, Evans AC (1994) Assessing the significance of focal activations using their spatial extent. Hum Brain Mapp 1:214-220.

Ghashghaei HT, Barbas H (2002) Pathways for emotion: interactions of prefrontal and anterior temporal pathways in the amygdala of the rhesus monkey. Neuroscience 115:1261-1279.

Ghashghaei HT, Hilgetag CC, Barbas H (2007) Sequence of information processing for emotions based on the anatomic dialogue between prefrontal cortex and amygdala. NeuroImage 34:905-923.

Gusnard DA, Akbudak E, Shulman GL, Raichle ME (2001) Medial prefrontal cortex and self-referential mental activity: relation to a default mode of brain function. Proc Natl Acad Sci USA 98:4259-4264.

Hamilton M (1959) The assessment of anxiety states by rating. Br J Med Psychol 32:50-55.

Hamilton M (1960) A rating scale for depression. J Neurol Neurosurg Psychiatry 23:56-62.

Jackson DC, Malmstadt JR, Larson CL, Davidson RJ (2000) Suppression and enhancement of emotional responses to unpleasant pictures. Psychophysiology 37:515-522.

Jackson DC, Mueller CJ, Dolski I, Dalton KM, Nitschke JB, Urry HL, Rosenkranz MA, Ryff CD, Singer BH, Davidson RJ (2003) Now you feel it, now you don't: frontal brain electrical asymmetry and individual differences in emotion regulation. Psychol Sci 14:612-617.

Jenkinson M, Bannister P, Brady M, Smith S (2002) Improved optimization for the robust and accurate linear registration and motion correction of brain images. NeuroImage 17:825-841.

Johnson SC, Baxter LC, Wilder LS, Pipe JG, Heiserman JE, Prigatano GP (2002) Neural correlates of self-reflection. Brain 125:1808-1814.

Johnstone T, Ores Walsh KS, Greischar LL, Alexander AL, Fox AS, Davidson RJ, Oakes TR (2006) Motion correction and the use of motion covariates in multiple-subject fMRI analysis. Hum Brain Mapp 27:779-788.

Kahneman D, Beatty J (1966) Pupil diameter and load on memory. Science 154:1583-1585.

Kasch KL, Rottenberg J, Arnow BA, Gotlib IH (2002) Behavioral activation and inhibition systems and the severity and course of depression. J Abnorm Psychol 111:589-597.

Keedwell PA, Andrew C, Williams SCR, Brammer MJ, Phillips ML (2005) A double dissociation of ventromedial prefrontal cortical responses to sad and happy stimuli in depressed and healthy individuals. Biol Psychiatry 58:495-503.

Kennedy SH, Evans KR, Kruger S, Mayberg HS, Meyer JH, McCann S, Arifuzzman AI, Houle S, Vaccarino FJ (2001) Changes in regional brain glucose metabolism measured with positron emission tomography after paroxetine treatment of major depression. Am J Psychiatry 158:899-905.
Lang PJ, Bradley MM, Cuthbert BN (2005) International affective picture system (IAPS): affective ratings of pictures and instruction manual. Technical Report A-6. Gainesville, FL: University of Florida.

Liotti M, Mayberg HS, McGinnis S, Brannan SL, Jerabek P (2002) Unmasking disease-specific cerebral blood flow abnormalities: mood challenge in patients with remitted unipolar depression. Am J Psychiatry 159:1830-1840.

Loewenfeld IE (1993) The pupil: anatomy, physiology, and clinical applications, Vol I. Ames, IA: Iowa State UP.

Maren S, Quirk GJ (2004) Neuronal signalling of fear memory. Nat Rev Neurosci 5:844-852.

Mayberg HS, Brannan SK, Mahurin RK, Jerabek PA, Brickman JS, Tekell JL, Silva JA, McGinnis S, Glass TG, Martin CC, Fox PT (1997) Cingulate function in depression: a potential predictor of treatment response. NeuroReport 8:1057-1061.

Meyer TJ, Miller ML, Metzger RL, Borkovec TD (1990) Development and validation of the Penn State worry questionnaire. Behav Res Ther 28:487-495.

Milad MR, Quirk GJ (2002) Neurons in medial prefrontal cortex signal memory for fear extinction. Nature 420:70-74.

Morris JS, Dolan RJ (2004) Dissociable amygdala and orbitofrontal responses during reversal fear conditioning. NeuroImage 22:372-380.

Ochsner KN, Bunge SA, Gross JJ, Gabrieli JD (2002) Rethinking feelings: an FMRI study of the cognitive regulation of emotion. J Cogn Neurosci 14:1215-1229.

Ochsner KN, Ray RD, Cooper JC, Robertson ER, Chopra S, Gabrieli JD, Gross JJ (2004) For better or for worse: neural systems supporting the cognitive down- and up-regulation of negative emotion. NeuroImage 23:483-499.

Ohira H, Nomura M, Ichikawa N, Isowa T, Iidaka T, Sato A, Fukuyama S, Nakajima T, Yamada J (2006) Association of neural and physiological responses during voluntary emotion suppression. NeuroImage 29:721-733.

Ongur D, Price JL (2000) The organization of networks within the orbital and medial prefrontal cortex of rats, monkeys and humans. Cereb Cortex 10:206-219.

Petersson KM, Nichols TE, Poline JB, Holmes AP (1999) Statistical limitations in functional neuroimaging II. signal detection and statistical inference. Philos Trans R Soc Lond B Biol Sci 354:1261-1281.

Pezawas L, Meyer-Lindenberg A, Drabant EM, Verchinski BA, Munoz KE, Kolachana BS, Egan MF, Mattay VS, Hariri AR, Weinberger DR (2005) 5-HTTLPR polymorphism impacts human cingulate-amygdala interactions: a genetic susceptibility mechanism for depression. Nat Neurosci 8:828-834.

Phan KL, Fitzgerald DA, Nathan PJ, Moore GJ, Uhde TW, Tancer ME (2005) Neural substrates for voluntary suppression of negative affect: a functional magnetic resonance imaging study. Biol Psychiatry 57:210-219.

Phelps EA, Delgado MR, Nearing KI, LeDoux JE (2004) Extinction learning in humans: role of the amygdala and vmPFC. Neuron 43:897-905.

Phillips ML, Drevets WC, Rauch SL, Lane R (2003) Neurobiology of emotion perception II: Implications for major psychiatric disorders. Biol Psychiatry 54:515-528.

Quirk GJ, Likhtik E, Pelletier JG, Pare D (2003) Stimulation of medial prefrontal cortex decreases the responsiveness of central amygdala output neurons. J Neurosci 23:8800-8807.

Rolls ET, Critchley HD, Mason R, Wakeman EA (1996) Orbitofrontal cortex neurons: role in olfactory and visual association learning. J Neurophysiol 75:1970-1981.

Schaefer SM, Jackson DC, Davidson RJ, Aguirre GK, Kimberg DY, Thompson-Schill SL (2002) Modulation of amygdalar activity by the conscious regulation of negative emotion. J Cogn Neurosci 14:913-921.

Siegle GJ, Steinhauer SR, Stenger VA, Konecky R, Carter CS (2003) Use of concurrent pupil dilation assessment to inform interpretation and analysis of fMRI data. NeuroImage 20:114-124.

Tomarken AJ, Davidson RJ, Wheeler RE, Doss RC (1992) Individual differences in anterior brain asymmetry and fundamental dimensions of emotion. J Pers Soc Psychol 62:676-687.

Urry HL, van Reekum CM, Johnstone T, Kalin NH, Thurow ME, Schaefer HS, Jackson CA, Frye CJ, Greischar LL, Alexander AL, Davidson RJ (2006) Amygdala and ventromedial prefrontal cortex are inversely coupled during regulation of negative affect and predict the diurnal pattern of cortisol secretion among older adults. J Neurosci 26:4415-4425.

van Reekum CM, Johnstone T, Urry HL, Thurow ME, Schaefer HS, Alexander AL, Davidson RJ (2007) Gaze fixations predict brain activation during the voluntary regulation of picture-induced negative affect. NeuroImage 36:1041-1055. 